\title{
Water vapor map of Mars near summer solstice using ground-based infrared spectroscopy
}

\author{
T. Encrenaz ${ }^{1}$, T. K. Greathouse ${ }^{2}$, B. Bézard ${ }^{1}$, T. Fouchet $^{1}$, F. Lefèvre ${ }^{3}$, F. Montmessin ${ }^{3}$, M. Bitner ${ }^{4}$, \\ A. Kruger ${ }^{5}$, M. J. Richter ${ }^{5}$, J. H. Lacy ${ }^{6}$, F. Forget ${ }^{7}$, and S. K. Atreya ${ }^{8}$ \\ 1 LESIA, Observatoire de Paris, CNRS, UPMC, UDD, 92195 Meudon, France \\ e-mail: therese.encrenaz@obspm.fr \\ 2 SWRI, Div. 15, San Antonio, TX 78228, USA \\ 3 UPMC, UVSQ, CNRS/INSU, LATMOS/IPSL, Boîte 102, 75005 Paris, France \\ ${ }^{4}$ STScI, Baltimore, MD 21218, USA \\ 5 Physics Department, University of California, Davis CA 95616, USA \\ 6 Department of Astronomy, University of Texas at Austin, TX 78712-1083, USA \\ 7 LMD/IPSL, UPMC, UVSQ, CNRS/INSU, 75231 Paris, France \\ 8 Department of Atmospheric, Oceanic and Space Sciences, University of Michigan, Ann Arbor, MI 48109-2143, USA
}

Received 18 December 2009 / Accepted 15 February 2010

\begin{abstract}
Ground-based spatial mapping of Mars provides a unique way to retrieve the global distribution of minor atmospheric species and to study transient phenomena or possible variations with the local hour. We have obtained an instantaneous map of water vapor on Mars near summer solstice $\left(\mathrm{Ls}=80^{\circ}\right)$ using the Texas Echelon Cross Echelle Spectrograph (TEXES) at the NASA Infrared Telescope Facility (IRTF) at Mauna Kea Observatory. Data have been obtained in the $1230-1245 \mathrm{~cm}^{-1}$ range $(\lambda=8.1 \mu \mathrm{m})$, with a spatial resolution of $1.1 \mathrm{arcsec}$ (after convolution) and a spectral resolution of $0.012 \mathrm{~cm}^{-1}\left(R=10^{5}\right)$. The map has been retrieved from the line depth of a weak HDO transition, compared with the line depth of a weak $\mathrm{CO}_{2}$ nearby transition. The TEXES map exhibits a strong maximum around the northern pole, as expected from previous observations and from climate model predictions. More interestingly, it shows longitudinal variations, both at high northern latitudes and at mid-latitudes, in close agreement with the predictions of the Global Climate Model developed at the Laboratoire de Meteorologie Dynamique (LMD GCM). The inferred water vapor mixing ratio is also in good agreement with the model predictions. The longitudinal variations at mid latitudes show a general enhancement toward the east. They do not seem to be due to the effect of local hour, but can be explained by dynamical effects generated by the topography. The map of surface temperatures, inferred from the continuum flux, is surprisingly different from the map expected from the climate models; the source of this discrepancy is still unclear.
\end{abstract}

Key words. planets and satellites: atmospheres - techniques: spectroscopic - planets and satellites: individual: Mars planets and satellites: surfaces

\section{Introduction}

The water vapor cycle of Mars has been extensively studied from space, both in the solar reflected spectrum and in the thermal regime. The thermal infrared range was used by the IRIS instrument aboard Mariner 9 (Conrath et al. 1973), then by TES aboard Mars Global Surveyor (Smith 2002, 2004) and more recently by PFS aboard Mars Express (Fouchet et al. 2007). In the reflected spectrum, both the MAWD experiment aboard Viking and SPICAM aboard Mars Express used the $1.38 \mu \mathrm{m} \mathrm{H}_{2} \mathrm{O}$ band (Farmer et al. 1977; Jakosky \& Haberle 1992; Fedorova et al. 2004, 2007) while OMEGA aboard Mars Express (Encrenaz et al. 2005, 2008; Melchiorri et al. 2007, 2009; Maltagliati et al. 2007, 2008) and CRISM aboard Mars Reconnaissance Orbiter (Smith et al. 2009) used the $2.6 \mu \mathrm{m} \mathrm{H}_{2} \mathrm{O}$ band, still in the reflected solar spectrum. All these analyses however have a common limitation, which is the lack of simultaneous mapping of the planet. The water vapor map is built from the co-addition of successive orbits which usually follow a meridian, so that, for a given time, there is no information about longitudinal or local time variations. To retrieve the longitudinal information, one needs to integrate over time. As a result, possible transient phenomena, or daily variations (from morning to evening) cannot be studied. There is actually an exception, when the orbital sequence crosses the polar region. In this case, morning and evening data can be acquired in a single orbit; however, this method is limited to the study of high latitude regions (Melchiorri et al. 2009).

In contrast, ground-based mapping using high spatial and spectral resolution makes the instantaneous mapping feasible (although the angular resolution is strongly degraded with respect to space observations). In addition, the spectral resolution of ground-based imaging spectrometers is typically much higher ( $R=10^{4}$ or more) than that of orbiting instruments, which makes possible the monitoring of the minor species on Mars (Encrenaz 2009). As an example, a ground-based monitoring of water vapor, at some specific locations, has been performed by Sprague et al. $(1996,2004)$ for over a decade.

We report here a ground-based observation of the water vapor global distribution near summer solstice $\left(L_{\mathrm{s}}=80^{\circ}\right)$. The interest for such a study was driven by the fact that, using OMEGA/Mars Express data obtained over the south pole just before the southern solstice $\left(L_{\mathrm{s}}=250-270^{\circ}\right)$ Melchiorri et al. (2009) reported some daily variability for southern latitudes 
higher than $60 \mathrm{~S}$, with an increase of a few precipitable microns between 4 am and $4 \mathrm{pm}$. As a possible explanation of this daily variation, it was suggested that the Martian regolith at high latitude might adsorb water during the night and desorb it during the day. The effect was found to be maximum slightly before southern solstice $\left(L_{\mathrm{S}}=260^{\circ}\right)$.

Using the Texas Echelon Cross Echelle Spectrograph (TEXES) instrument at the Infrared Telescope Facility (IRTF) at Mauna Kea Observatory, we have observed the equivalent situation in the northern hemisphere, shortly before northern solstice $\left(L_{\mathrm{s}}=80^{\circ}\right)$. The geometry allowed us to map the whole disk of Mars with latitudes ranging from 40S to $80 \mathrm{~N}$, which gave us the opportunity to investigate the possible daily variation at midlatitudes. Our study, unfortunately, was limited by the low spatial resolution of the data, due to the small size of the Martian disk; still our water vapor map could be compared with GCM predictions. The present article describes this analysis. The main conclusion is that, for $L_{\mathrm{s}}=80^{\circ}$ and for northern latitudes lower than $60 \mathrm{~N}$, the observed water vapor map is in good agreement with the climate model predictions, which do not include the absorption/desorption mechanism. Longitudinal variations of the water vapor mixing ratio are observed at mid-latitudes, but they can be explained by dynamical motions due to topography effects. Observations are presented in Sect. 2. Section 3 describes the retrieval method of the water vapor mixing ratio. Our results are discussed in Sect. 4.

\section{Observations and data analysis}

Since 2001, we have been mapping the Martian disk with the TEXES instrument at IRTF, with the prime objective of detecting and mapping hydrogen peroxide (Encrenaz et al. 2002). The spectral range was $1230-1245 \mathrm{~cm}^{-1}$, corresponding to a wavelength of $8.1 \mu \mathrm{m} . \mathrm{H}_{2} \mathrm{O}_{2}$ was detected in 2003 and later observed in 2005 and 2008, allowing us to start monitoring its seasonal variability (Encrenaz et al. 2004, 2008, 2009). In addition, we have been using weak transitions of HDO to monitor the water vapor simultaneously (Encrenaz et al. 2005, 2008, 2009).

The observing run described in this paper was performed on May-June 2008. A marginal detection of $\mathrm{H}_{2} \mathrm{O}_{2}$ was achieved, leading to a mean mixing ratio of $10 \mathrm{ppb}$ over the disk (Encrenaz et al. 2009). A map of water vapor was obtained on June 1, 2008 between 03:00 and 04:00 UT. The areocentric longitude $L_{\mathrm{s}}$ was $80^{\circ}$, i.e. slighly before summer solstice. The mean latitude and longitude of the disk center were $19 \mathrm{~N}$ and $135 \mathrm{E}$ respectively; the subsolar point was located at $25 \mathrm{~N}$ and $170 \mathrm{E}$. The terminator was situated on the morning side and the illumination factor was 92 percent. The local hour at the center of the disk was 10:00 am. The diameter of Mars was 5 arcsec; the small size of the Martian disk was a significant limitation, as compared to our previous runs. The radial velocity was $14 \mathrm{~km} \mathrm{~s}^{-1}$, corresponding to a doppler shift of $-0.058 \mathrm{~cm}^{-1}$ at $1240 \mathrm{~cm}^{-1}$.

Data were recorded in the $1232-1237 \mathrm{~cm}^{-1}$ range, with a spectral resolution of $0.012\left(R=10^{5}\right)$. The observing sequence was the same as for previous runs on Mars: the $1.1 \times 8 \operatorname{arcsec}^{2}$ slit was oriented along the celestial $N-S$ axis (less than 2 degrees off the Martian central meridian) and moved from west to east by 0.5 arcsec steps. Each map was acquired in about 5 min and about 10 individual maps were recorded. These maps were co-added by superimposing the maximum flux measured in the continuum. The individual pixel size was 0.368 arcsec and the spatial resolution, after convolution over 3 pixel elements, was $1.1 \mathrm{arcsec}$. The data reduction and radiance calibration are described in Encrenaz et al. (2004, 2005).

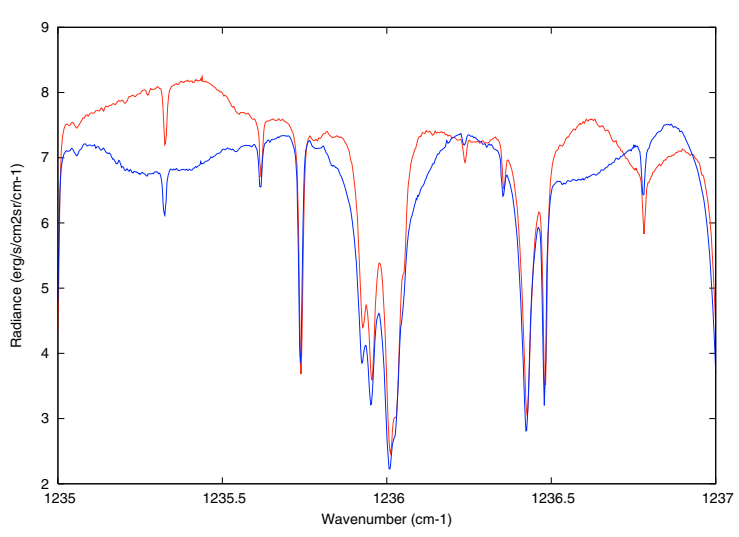

Fig. 1. Two spectra of Mars recorded by the TEXES instrument, integrated over the northern hemisphere $(30 \mathrm{~N}-50 \mathrm{~N}$, red curve) and the equatorial region ( $5 \mathrm{~N}-15 \mathrm{~S}$, blue curve). It can be seen that the Martian $\mathrm{HDO}$ transition at $1236.237 \mathrm{~cm}^{-1}$ is slightly stronger in the northern hemisphere. Martian $\mathrm{CO}_{2}$ transitions appear in particular at 1235.325 and $1235.617 \mathrm{~cm}^{-1}$. The strong bands around 1236 and $1236.5 \mathrm{~cm}^{-1}$ are due to telluric absorption.

Figure 1 shows a part of the spectrum between 1235 and $1237 \mathrm{~cm}^{-1}$, which includes the HDO transition used in this study as well as several $\mathrm{CO}_{2}$ transitions of different intensities. The strong absorption bands around 1236 and $1236.5 \mathrm{~cm}^{-1}$ are due to terrestrial absorption by $\mathrm{CH}_{4}$ and $\mathrm{H}_{2} \mathrm{O}$. Two spectra are shown, integrated respectively over the northern hemisphere $(30 \mathrm{~N}-50 \mathrm{~N})$ and the equatorial region $(5 \mathrm{~N}-15 \mathrm{~S})$. It can be seen that the continuum between the lines shows strong fluctuations, which we attribute to instrumental effects associated to the poor seeing conditions (the observations took place between 17:00 and 18:00 local time).

The HDO transition used in our study has a rest frequency of $1236.295 \mathrm{~cm}^{-1}$ and is observed, due to the doppler shift, at a frequency of $1236.237 \mathrm{~cm}^{-1}$. This transition was used during our 2005 run. The $1239.996 \mathrm{~cm}^{-1}$ transition used during our 2003 run could not be used this time because, due to the negative doppler shift, it fell inside a terrestrial water absorption at $1239.94 \mathrm{~cm}^{-1}$. Several $\mathrm{CO}_{2}$ transitions of weak intensities can be used for comparison. Two transitions are well isolated and have been used in this study: the $1235.383 \mathrm{~cm}^{-1}$ transition, shifted to $1235.325 \mathrm{~cm}^{-1}\left(I=0.420 \times 10^{-26} \mathrm{~cm} \mathrm{molec}^{-1}, E=67.0 \mathrm{~cm}^{-1}\right)$ and the $1235.675 \mathrm{~cm}^{-1}$ doublet, shifted to $1235.617 \mathrm{~cm}^{-1}(I=$ $0.903 \times 10^{-26} \mathrm{~cm} \mathrm{molec}^{-1}$ and $E=673.4 \mathrm{~cm}^{-1}$ for each component of the doublet). Both led to identical results.

Figure 2 shows the distribution of the continuum flux, obtained as a mean value of the continum at 4 frequencies, chosen on each side of the HDO transition and the $\mathrm{CO}_{2}$ transitions respectively. We have checked that the map is unchanged when different sets of continuum values are used. It can be seen that the image is blurred, which is due to a combination of the poor seeing and the small size of the Martian disk. The continuum flux is the thermal blackbody radiation corresponding to the surface temperature multiplied by the dust emissivity (typically above 0.9 at this wavelength). Surprisingly, the maximum of the flux continuum is located on the left side of the subsolar point (at about 11:00 local time) while it would have been expected on the right side. This discrepancy is discussed in more detail below (Sect. 4.1). 


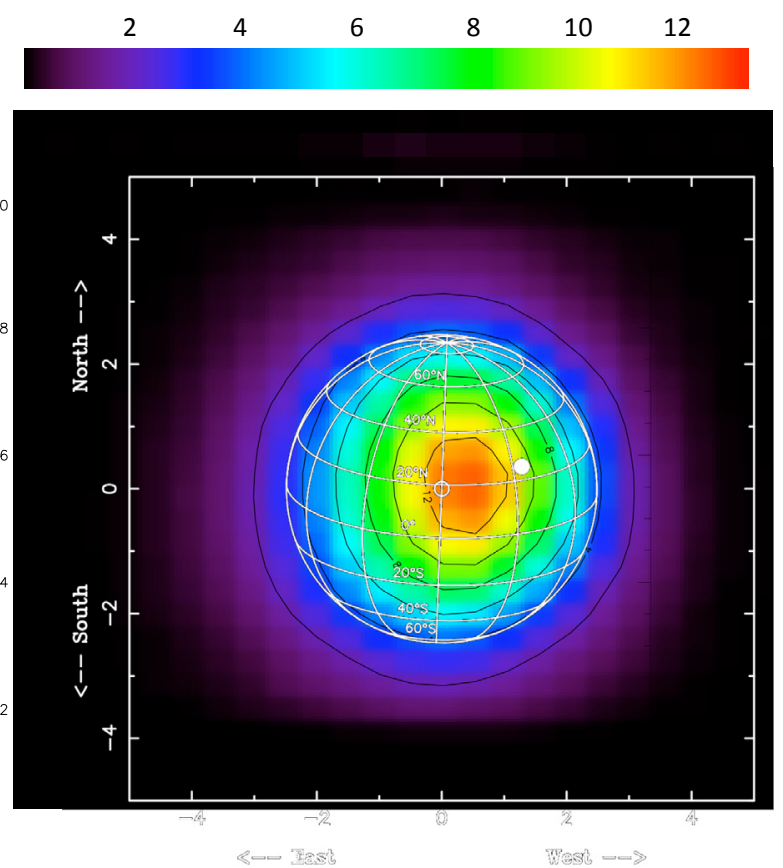

Fig. 2. The TEXES map of the continuum radiance averaged over 4 frequencies around $1236 \mathrm{~cm}^{-1}$. The size of the Martian disk is 5 arcsec. The longitude of the central meridian is $135 \mathrm{E}$. The white dot corresponds to the sub-solar point.Units are in arcsec in the celestial coordinates.

\section{Retrieval of the water vapor mixing ratio}

Our retrieval method has been described in previous publications (Encrenaz et al. 2004, 2005). Spectroscopic data are taken from the GEISA data bank (Jacquinet-Husson et al. 2008). Weak $\mathrm{CO}_{2}$ isotopic transitions have been added following the work of Rothman (1986) and Toth (1985). The two spectra shown in Fig. 1 have been modelled using surface and atmospheric parameters extracted from the Mars Global Climate Model (GCM) of the Laboratoire de Meteorologie Dynamique (LMD) corresponding to the conditions of our observation. For the northern and southern hemispheres, we used similar temperature profiles. In agreement with the surface temperature map derived from the continuum measurements (Figs. 2 and 10), we used a surface temperature of $235 \mathrm{~K}$. The atmospheric temperature was $215 \mathrm{~K}$ at $z=0,175 \mathrm{~K}$ at $z=22 \mathrm{~km}, 165 \mathrm{~K}$ at $z=38 \mathrm{~km}$, and $155 \mathrm{~K}$ at $z=50 \mathrm{~km}$ and above. The surface pressure was $7.0 \mathrm{mbar}$ in the northern hemisphere and 6.5 mbar in the southern one. The airmass was 1.7 for both spectra.

As in our previous studies, we have assumed for the Martian atmosphere a constant $\mathrm{HDO} / \mathrm{H}_{2} \mathrm{O}$ mixing ratio equal to 5 times the terrestrial value, as derived by Krasnopolsky et al. (1997). However this assumption may be incorrect, due to possible fractionation effects associated to water condensation and sublimation (Fouchet \& Lellouch 2007) or photodissociation. Montmessin et al. (2005) have calculated the expected D/H ratio on Mars as a function of latitude and season. According to their study, fractionation effects are maximum above the polar caps, where $\mathrm{D} / \mathrm{H}$ in the vapor phase may be reduced by a factor 2 , because they mainly depend upon condensation effects. Near summer solstice $\left(L_{\mathrm{s}}=80^{\circ}\right), \mathrm{D} / \mathrm{H}$ is expected to be between 4.8 and 5 times the terrestrial value for latitudes ranging from 0 to $90 \mathrm{~N}$. The associated uncertainty should thus be less than 4 percent in this region. Between 0 and $30 \mathrm{~S}$, the Martian D/H is expected to range between 4.5 and 4.8 times the terrestrial value

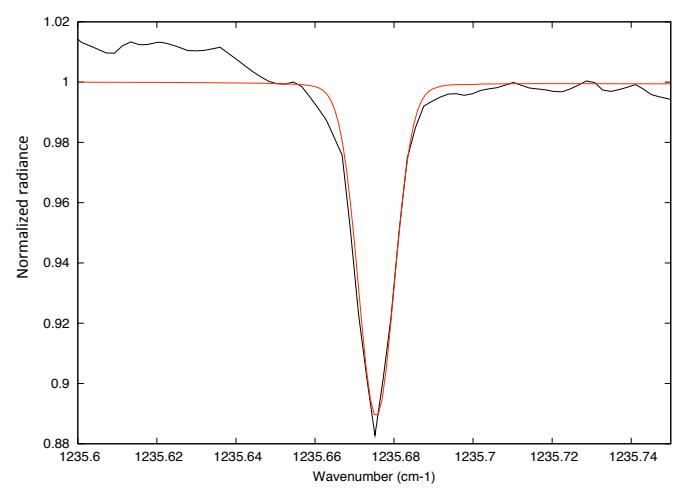

Fig. 3. Black line: The TEXES $\mathrm{CO}_{2}$ line in the northern spectrum (same as Fig. 1). Blue line: The synthetic spectrum calculated with the GCM parameters corresponding to the observation. The airmass is 1.7.

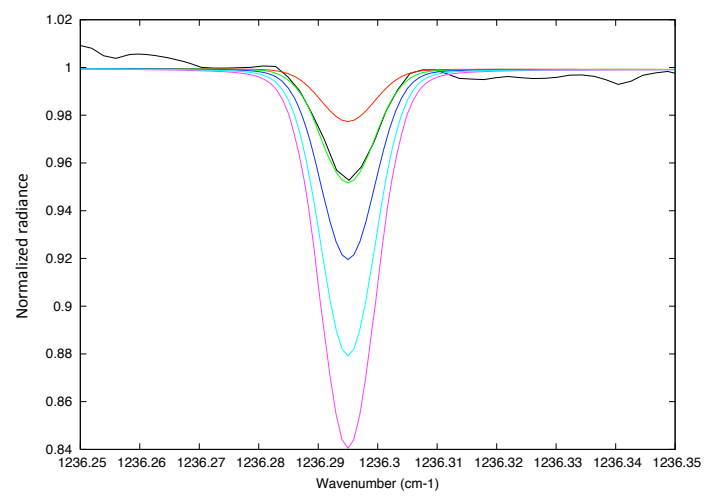

Fig. 4. Black line: The TEXES HDO line in the northern spectrum (same as Fig. 1). Color lines: Synthetic spectra calculated with the GCM parameters corresponding to the observation. The airmass is 1.7. From top to bottom; $\left[\mathrm{H}_{2} \mathrm{O}\right]=100,250,500,750,1000 \mathrm{ppm}$.

(Montmessin et al. 2005). Consequently, our water vapor measurements at southern latitudes would thus be underestimated by about ten percent. However, simultaneous observations of HDO and $\mathrm{H}_{2} \mathrm{O}$ performed by Mumma et al. (2003) and Novak et al. (2007) seem to indicate different results. Novak et al. (2007) report variations of $\mathrm{D} / \mathrm{H}$ with time and season, with a $\mathrm{D} / \mathrm{H}$ higher in the southern hemisphere than in the north. So the uncertainty on the actual $\mathrm{D} / \mathrm{H}$ ratio, although difficult to quantify, might be higher than 10 percent.

Figures 3 and 4 show, for the northern spectrum, the simultaneous fit of the $\mathrm{CO}_{2}$ transition at $1235.675 \mathrm{~cm}^{-1}$ (Fig. 3) and the $\mathrm{H}_{2} \mathrm{O}$ transition at $1236.295 \mathrm{~cm}^{-1}$ (Fig. 4) using the parameters mentioned above. Figure 4 shows that the best fit is obtained for a $\mathrm{H}_{2} \mathrm{O}$ mixing ratio of $250 \mathrm{ppm}$, which corresponds to a water vapor column density of $15 \mathrm{pr}-\mu \mathrm{m}$ assuming a uniform mixing ratio. Figures 5 and 6 show the same analysis for the southern spectrum. In this case, the best fit is obtained for an $\mathrm{H}_{2} \mathrm{O}$ mixing ratio of $125 \mathrm{ppm}$, which corresponds to a water vapor mixing ratio of $7.4 \mathrm{pr}-\mu \mathrm{m}$, still assuming a constant mixing ratio.

As in the case of our previous studies, we have chosen a simple method to infer the water vapor mixing ratio over the Martian disk, by using the ratio of the line depth ratios of 2 weak transitions, $\mathrm{HDO}$ and a nearby $\mathrm{CO}_{2}$ transition. This method has the advantage of reducing the effects associated with geometrical and atmospheric parameters (airmass, dust opacity...). An analysis of the uncertainties associated with this method has been given in Encrenaz et al. (2008). It has been shown that our method can induce a significant error (as high as 30 percent in some cases 


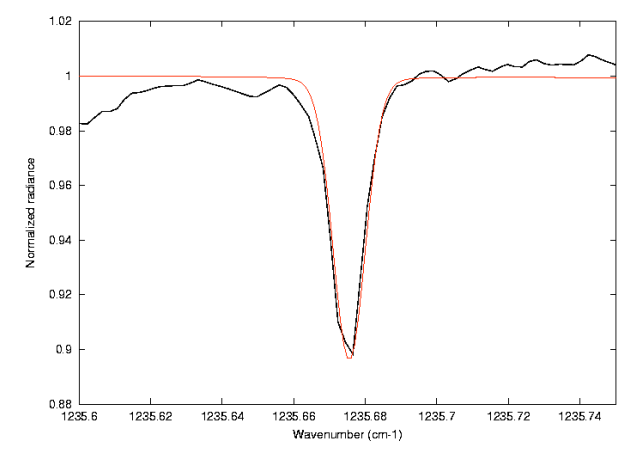

Fig. 5. Black line: The TEXES $\mathrm{CO}_{2}$ line in the southern spectrum (same as Fig. 1). Blue line: the synthetic spectrum calculated with the GCM parameters corresponding to the observation. The airmass is 1.7 .

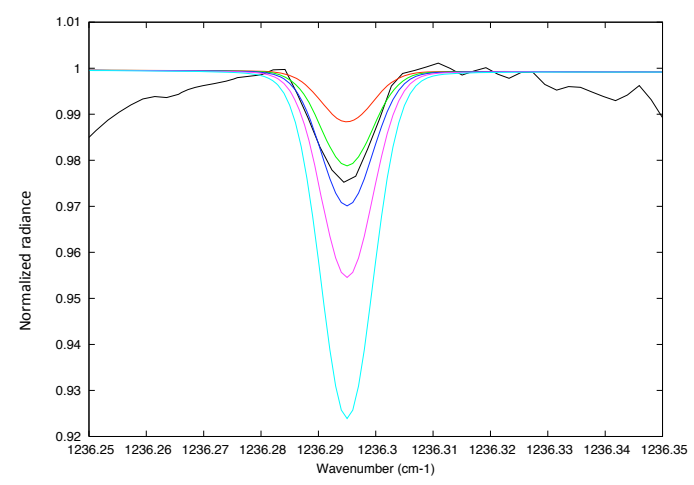

Fig. 6. Black line: The TEXES HDO line in the southern spectrum (same as Fig. 1). Color lines: Synthetic spectra calculated with the GCM parameters corresponding to the observation. The airmass is 1.7. From top to bottom: $\left[\mathrm{H}_{2} \mathrm{O}\right]=50,100,150,250,500 \mathrm{ppm}$.

for the highest airmass). In the case of HDO, the typical error was found to be in the range of 5 to 10 percent.

Figures 7 and 8 show the maps of the line depths for the $\mathrm{CO}_{2}$ transition at $1235.675 \mathrm{~cm}^{-1}$ and the HDO transtion at $1236.295 \mathrm{~cm}^{-1}$ respectively. A similar $\mathrm{CO}_{2}$ map is obtained with the $1235.383 \mathrm{~cm}^{-1}$ transition. Figure 9 a shows the line depth ratio of the $\mathrm{HDO}$ transition divided by the $\mathrm{CO}_{2} 1235.675 \mathrm{~cm}^{-1}$ transition.

\section{Discussion}

\subsection{Surface temperature}

A map of the surface temperature can be retrieved from the TEXES continuum map (Fig. 2) assuming a given emissivity at $1240 \mathrm{~cm}^{-1}$ (Encrenaz et al. 2008). Figure 10a shows the inferred surface temperature map, taking a mean emissivity of 0.95 . For comparison, Fig. 10b shows the surface temperature for $L_{\mathrm{S}}=$ $80^{\circ}$, as predicted by the LMD GCM (Forget et al. 1999, 2006; Lefevre et al. 2008). There is a striking discrepancy between the two figures, with the expected local maximum on the evening side (on the right side of the SSP) being absent in the TEXES map. Apart from this big difference, the two maps are globally consistent within $15 \mathrm{~K}$. In particular the local maximum above

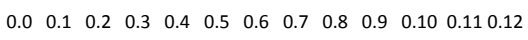

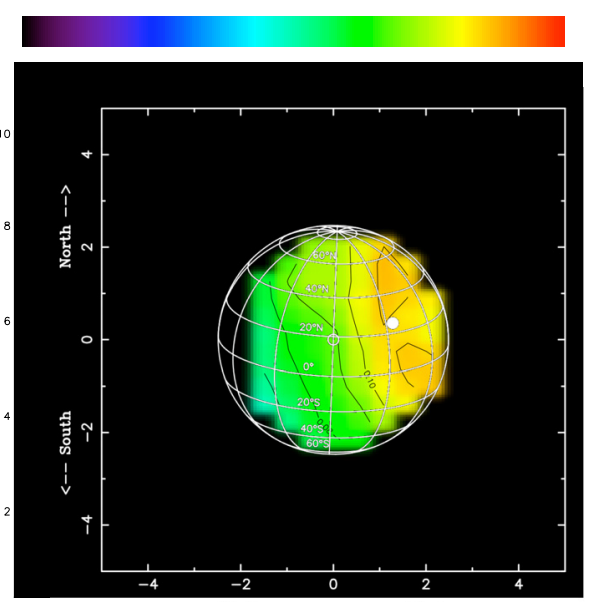

Fig. 7. Map of the line depth of the $\mathrm{CO}_{2}$ transition at $1235.675 \mathrm{~cm}^{-1}$. The SSP is indicated with a white dot.

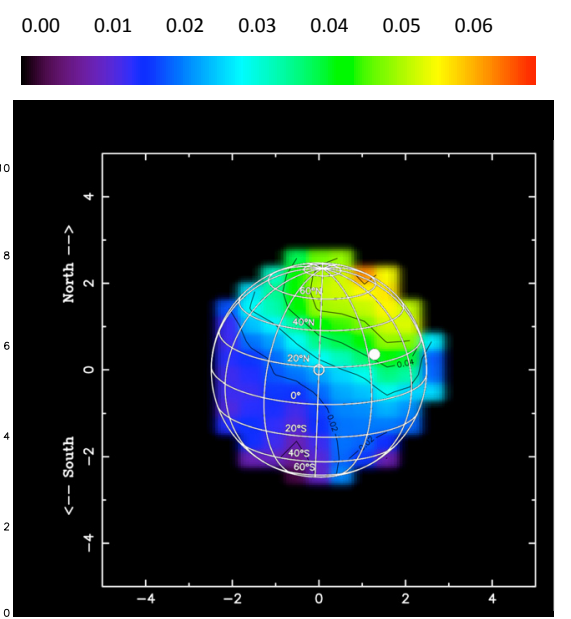

Fig. 8. Map of the line depth of the HDO transition at $1236.295 \mathrm{~cm}^{-1}$. The SSP is indicated with a white dot.

Elysium has a temperature of $245 \mathrm{~K}$, consistent with the GCM predictions.

What could be the reason for the discrepancy between the observations and the model? We reported a similar result regarding our 2005 run $\left(L_{\mathrm{s}}=332^{\circ}\right.$; Encrenaz et al. 2008). The temperature maximum had the expected intensity but was shifted southward with regard to the expected position; the possible presence of dust was invoked as a tentative explanation of the discrepancy. In 2003, in contrast, the TEXES temperature map $\left(L_{\mathrm{s}}=206^{\circ}\right)$ was in good agreement with the GCM (Encrenaz et al. 2005).

We first wondered if the TEXES temperature map, and in particular the absence of strong maximum on the evening side, could result from the presence of a significant amount of aerosol (dust or ice cloud) above this region. The aerosols would have to be located over the region centered around $(30 \mathrm{~N}, 180 \mathrm{E})$ which corresponds to Amazonis Planitia. In order to lower the surface temperature from $280 \mathrm{~K}$ (as predicted by the GCM) to $230 \mathrm{~K}$ (as observed by TEXES), an opacity as high as 1.4 is required. Because of the high airmass, the nadir opacity would have to be in the range $0.4-0.7$. Such dust abundances are not uncommon, although not expected at this time in the season. Indeed, THEMIS observations performed at the same period show that, for $L_{\mathrm{s}}=80^{\circ}$, the depth optical depth at $8 \mu \mathrm{m}$, integrated over longitudes, is less than 0.1 (Smith 2009). The large global-scale 
T. Encrenaz et al.: Water vapor mapping on Mars near summer solstice
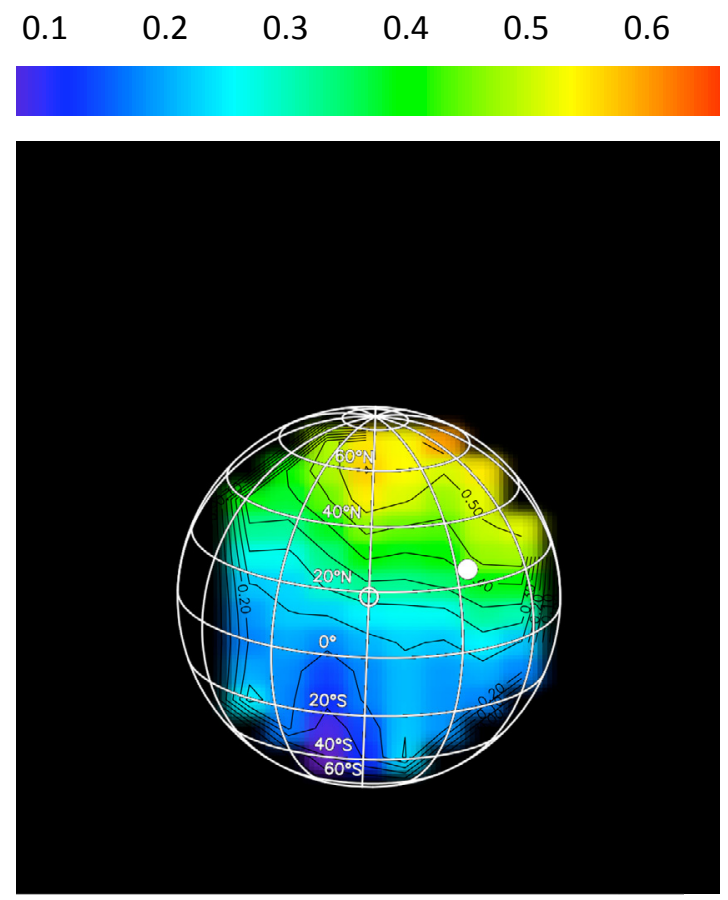

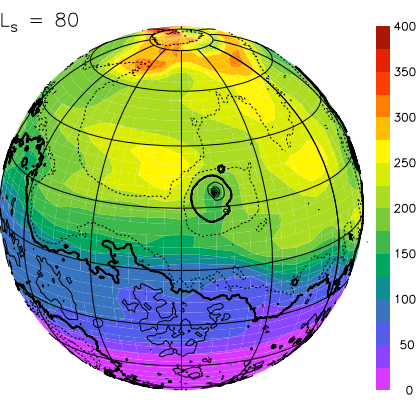

$\mathrm{H}_{2} \mathrm{O}$ mean mixing ratio (ppmv)

Fig. 9. a) Left side: map of the $\mathrm{HDO} / \mathrm{CO}_{2}$ line depth ratio. A line depth ratio of 0.5 corresponds to a $\mathrm{H}_{2} \mathrm{O}$ mixing ratio of 250 ppm (see Figs. $3 \mathrm{a}$,b). The SSP is indicated with a white dot; b) right side: the $\mathrm{H}_{2} \mathrm{O}$ mixing ratio map predicted by the GCM for the conditions of our observations.

$\begin{array}{lllll}190 & 200 \quad 210 \quad 220 \quad 230 \quad 240\end{array}$
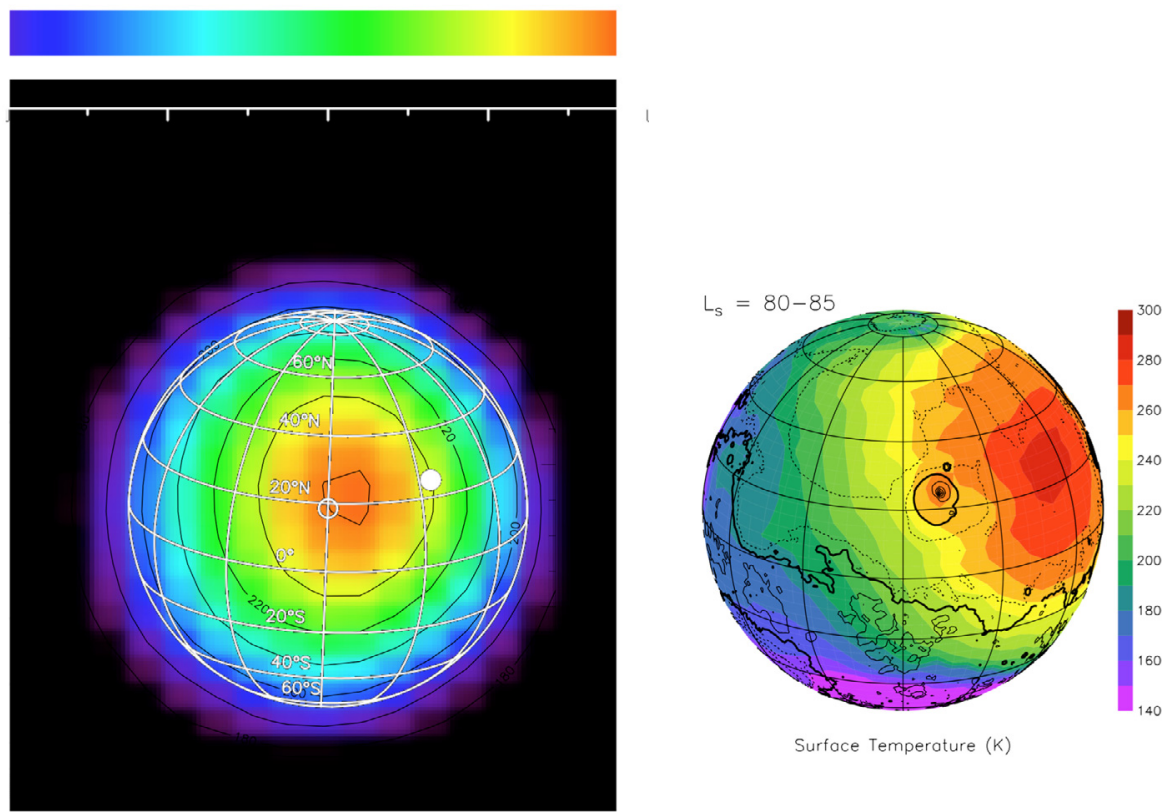

Surface Temperature ( $K$ )

Fig. 10. a) Left side: the TEXES map of the surface temperature retrieved from the continuum map at $1235 \mathrm{~cm}^{-1}$ (Fig. 2), assuming an emissivity of 0.95 . The white dot shows the position of the SSP. The longitude of the disk center is 135E. b) Right side: the surface temperature map predicted by the LMD GCM for the conditions of our observations.

shift in the apparent brightness temperature would require dense dust (or ice) clouds over a large regional extent with clear skies over the rest of the planet. Large regional dust storms of this magnitude are unknown at this Martian season, and were not observed by any Martian spacecraft at the time of the TEXES observations. Thus, this hypothesis seems very unlikely.
The map of the $\mathrm{CO}_{2}$ line depth (Fig. 7) is not directly interpretable in terms of dust content, because the $\mathrm{CO}_{2}$ line depth is a function of several parameters (surface pressure, surface temperature, atmospheric thermal profile, topography, air mass). Still, it can be seen from Fig. 7 that the $\mathrm{CO}_{2}$ line depth increases from morning to afternoon. This is what is expected 
if the surface temperature increases more rapidly than the atmosphere from morning to afternoon, as illustrated by the GCM map of Ts (Fig. 11). Thus, the map of the $\mathrm{CO}_{2}$ line depth appears, to first order, consistent with the model expectations.

We then investigated if the puzzling aspect of the TEXES continuum map could be of instrumental origin. As illustrated in Fig. 1, the TEXES continuum is affected by wiggles, most probably due to Fabry-Perot finging in the circular variable filter. These effects may be about $10-20$ percent (Fig. 1), which is still not sufficient to explain the discrepancies in the observed and predicted brightness temperatures. A more significant effect is probably the poor spatial resolution of our data. As the slit width is three times the pixel size, the observed continuum value at the limb position is reduced by a factor 3 as compared to the true continuum value. As a result, the high temperature contrast expected near the evening limb is significantly reduced. As mentioned above, the blurring effect is further enhanced with the poor seeing. This convolution effect, however, cannot explain the large difference between the observed and predicted surface temperatures, so this remains an open question. In any case, we conclude that the continuum map may not be a reliable indicator of the surface temperature. In contrast, the $\mathrm{CO}_{2}$ and $\mathrm{H}_{2} \mathrm{O}$ line depths, and thus the water vapor map, are not affected by the uncertainty in the continuum level.

\subsection{Water vapor mixing ratio}

Figure $9 \mathrm{~b}$ shows the expected water vapor mixing ratio as derived from the GCM for the conditions of our observation. This map is to be compared to Fig. 9a which shows the $\mathrm{HDO} / \mathrm{CO}_{2}$ line depth ratio. This map cannot be directly converted in terms of the $\mathrm{H}_{2} \mathrm{O}$ mixing ratio, because this conversion implies the choice of surface and atmospheric parameters (in particular surface pressure and temperature) which vary over the disk. Thus, as pointed above, Fig. 9 only gives a qualitative estimate of the water vapor mixing ratio. As shown in Figs. 3 and 4, a line depth ratio of 0.5 corresponds to a $\mathrm{H}_{2} \mathrm{O}$ mixing ratio of $250 \mathrm{ppm}$. There is a good agreement between the estimates shown in Fig. 9a and the GCM predictions (Fig. 9b).

An interesting feature of the TEXES map is the slight increase of the water vapor mixing ratio from western to eastern longitudes (i.e. from morning to evening), observed over all northern latitudes. This trend is also predicted by the GCM model (Fig. 9b). To first order, we can state that the spatial distribution of the water vapor mixing ratio is well reproduced by the climate model. The GCM is calculated on the basis of dynamical effects associated with the topography of the Martian globe. The good agreement between the GCM and the TEXES data shows that, at northern latitudes lower than $60 \mathrm{~N}$, there is no need for any new mechanism like regolith desorption to account for the data. Because of the limited spatial resolution of our data, no conclusion can be derived at higher latitudes from the present analysis. Higher spatial resolutions can be achieved in the future when Mars is closer to opposition, and/or if TEXES is used on the GEMINI telescope.

Acknowledgements. We thank the IRTF staff for the support of TEXES observations. Observations with TEXES were supported by NSF Grants AST-0607312 for J.H.L. and AST-0708074 for MJR. T.K.G. acknowledges support by NASA Grant NNX08AW33G S03 for data reduction. T.E. and B.B. acknowledge support from CNRS, and TF aknowledges support from UPMC. We are grateful to M.D. Smith for helpful comments regarding this paper.

\section{References}

Encrenaz, T. 2009, Earth, Moon and Planets, 105, 127

Encrenaz, T., Bézard, B., Greathouse, T. K., et al. 2004, Icarus, 170, 424

Encrenaz, T., Bézard, B., Owen, T., et al. 2005, Icarus, 179, 43

Encrenaz, T., Greathouse, T. K., Richter, M. J., et al. 2008, Icarus, 195, 547

Encrenaz, T., Greathouse, T. K., Bitner, M., et al. 2009, ESLAB Conference International Conference on Comparative Planetology, Noordwijk, May, 144 Farmer, C. B., Davis, D. W., Holland, A. L., et al. 1977, J. Geophys. Res., 82, 4225

Fedorova, A. A., Rodin, A. V., \& Baklanova, I. V. 2004, Icarus, 171, 54

Fedorova, A. A., Korablev, O., Bertaux, J.-L., et al. 2007, BAAS, 39, 31.03

Forget, F., Hourdin, F., Fournier, R., et al. 1999, J. Geophys. Res. 104, 24155

Forget, F., Montabone, L., \& Lebonnois, S. 2006, Second International Workshop on Mars Atmosphere Modelling and Observations, Granada, Feb. 27-Mar. 3

Fouchet, T., Lellouch, E., Ignatiev, N., et al. 2007, Icarus, 190, 32

Jacquinet-Husson, N., Scott, N., Chedin, A., et al. 2008, J. Quant. Spectr. Rad. Trans., 109, 1043

Jakosky, B. M., \& Haberle, R. M. 1992, in Mars, ed. H. H. Kieffer, et al. (University of Arizona Press)

Lacy, J. H., Richter, M. J., Greathouse, T. K., et al. 2002, Pub. Astron. Soc. Pacific, 114, 153

Lefevre, F., Bertaux, J.-L., Clancy, R. T., et al. 2008, Nature, 454, 971

Maltagliati, L., Titov, D., Encrenaz, T., et al. 2007, Proc. European Mars Science and Exploration Conf., Noordwijk, 12-16 November, 262

Maltagliati, L., Titov, D., Encrenaz, T., et al. 2008, Icarus, 194, 53

Melchiorri, R., Encrenaz, T., Fouchet, T., et al. 2007, Plan. Space Sci., 55, 333

Melchiorri, R., Encrenaz, T., Drossart, P., et al. 2009, Icarus, 201, 102

Mumma, M., Novak, R. E., Di Santi, M. A., et al. 2003, in Sixth Conference on the Martian amosphere, Pasadena, CA, July 20-25

Montmessin, F., Fouchet, T., \& Forget, F. 2004, J. Geophys. Res., 110 (E3)

Novak, R. E., Mumma, M. J., Villanueva, G., et al. 2007, BAAS, 39

Rothman, L. S. 1986, Appl. Opt. 25, 1795

Smith, M. D. 2002, J. Geophys. Res. 107, E11, 5115

Smith, M. D. 2004, Icarus, 167, 148

Smith, M. D. 2009, Icarus, 202, 444

Smith, M. D., Wolff, M. J., Clancy, R. T., \& Murchie, S. L. 2009, J. Geophys. Res. 114, E00, D03

Sprague, A. L., Hunten, D. M., Hill, R. E., et al. 1996, J. Geophys. Res., 101, 23229

Sprague, A. L., Boynton, W. V., Kerry, K. E., et al. 2004, Science, 306, 1364

Toth, R. A. 1985, Appl. Opt. 24, 261 